Supporting information

\title{
A Mono-Targeting Peptidic Network Antibody Inhibits More Receptors for Anti-Angiogenesis
}

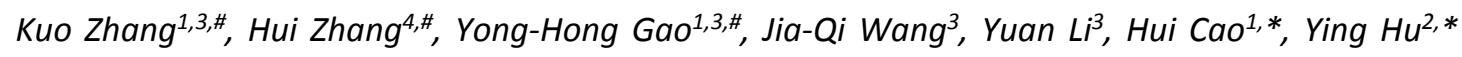
and Lei Wang ${ }^{3, *}$

${ }^{1}$ Department of Materials Physics and Chemistry, School of Materials Science and Engineering, University of Science and Technology Beijing, No. 30 Xueyuan Road, Beijing, 100083, China

2 Shanghai Jiao Tong University Affiliated Sixth People's Hospital, 600, Yishan Road, Shanghai, 200233, China

${ }^{3}$ CAS Center for Excellence in Nanoscience, CAS Key Laboratory for Biomedical Effects of Nanomaterials and Nanosafety, National Center for Nanoscience and Technology (NCNST), No. 11 Beiyitiao, Zhongguancun, Beijing, 100190, China

${ }^{4}$ Shanghai Jiao Tong University School of Medicine, 227 Chongqing South Road, Shanghai, 200025, China 
a)

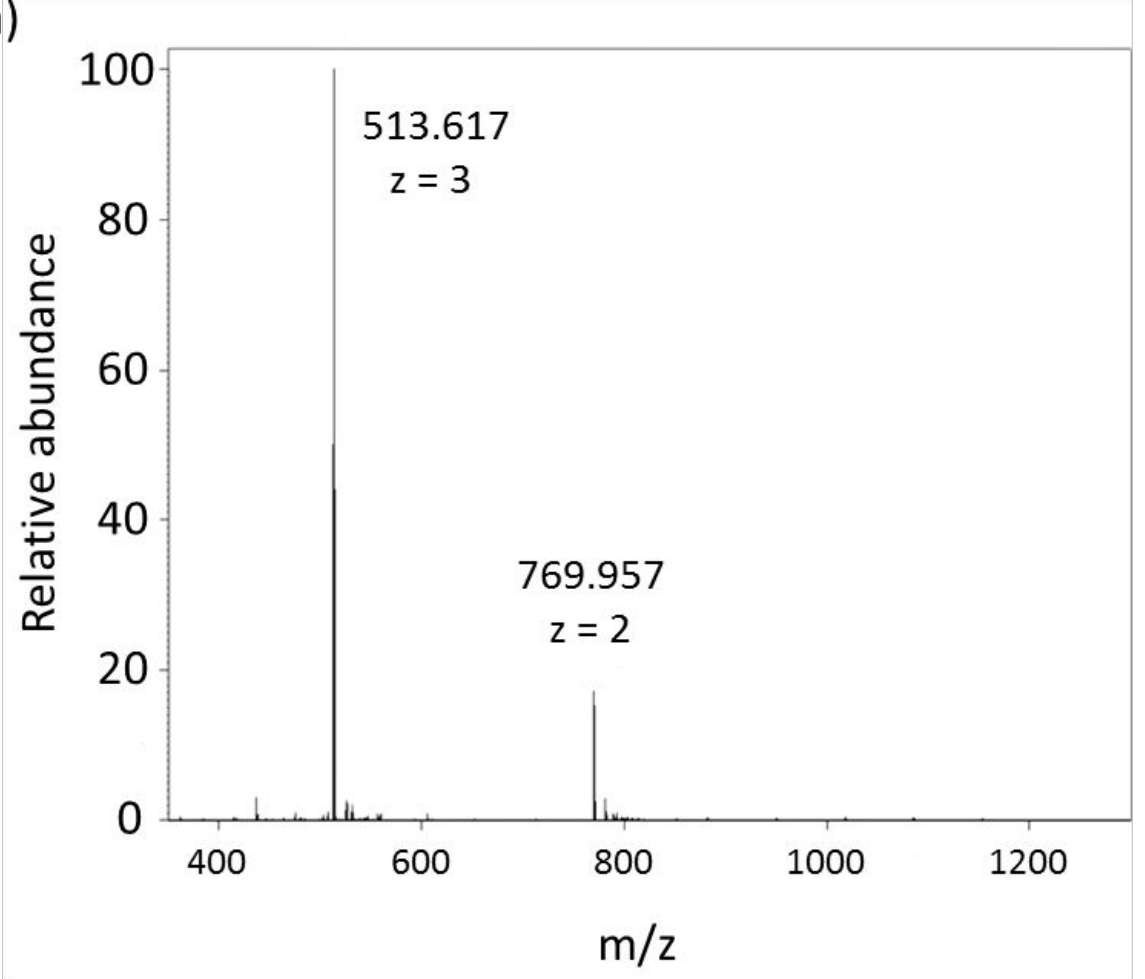

b)

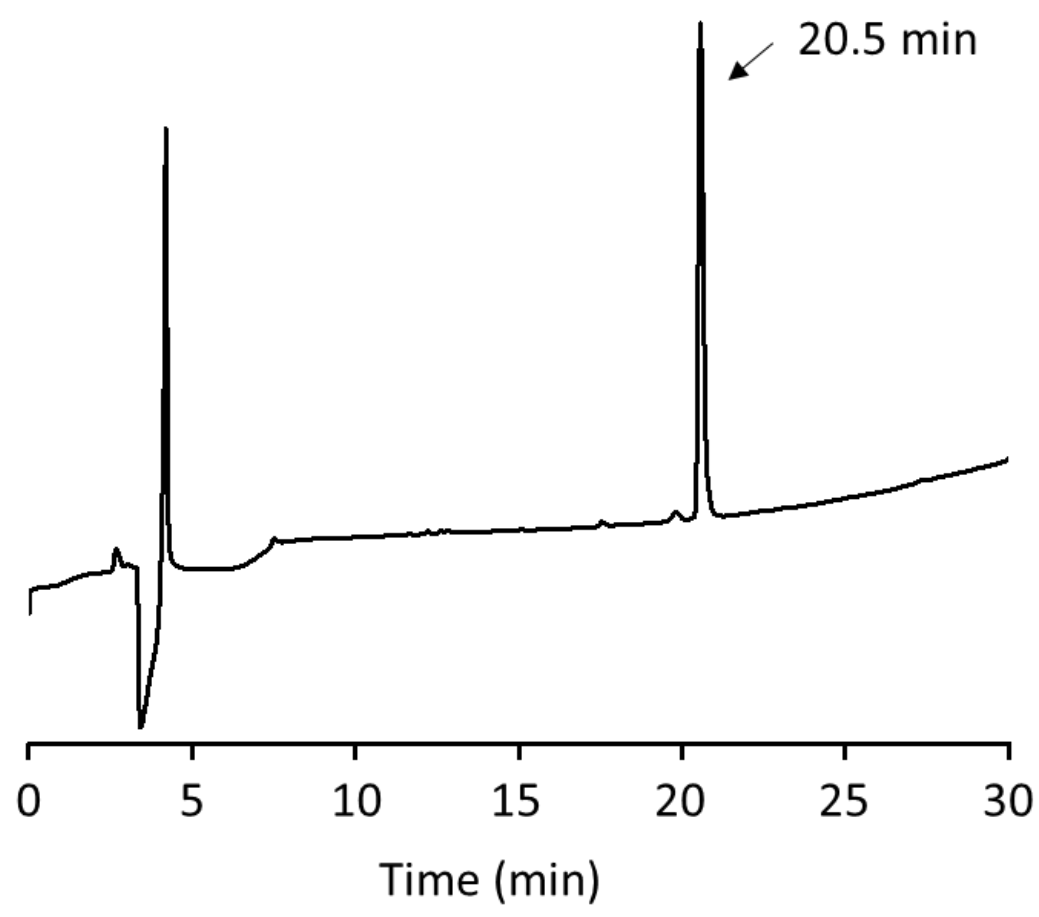

Fig. S1. Characterization of LAFH by a) Electrospray Ionization Mass Spectrometer and b) High Performance Liquid Chromatography (HPLC). 
a)

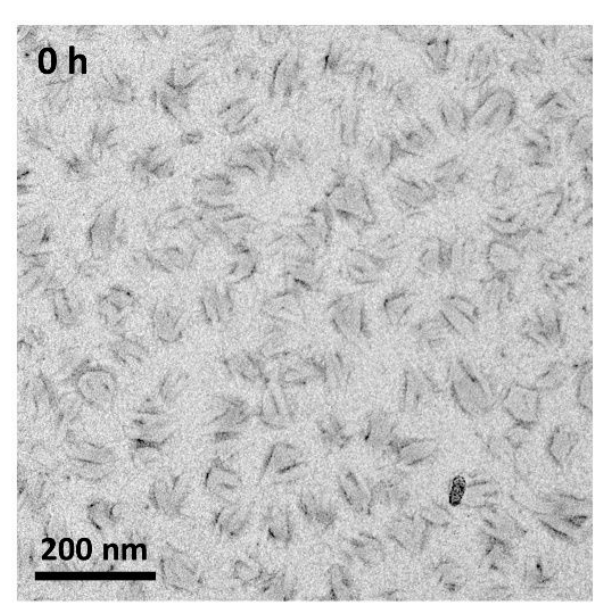

c)

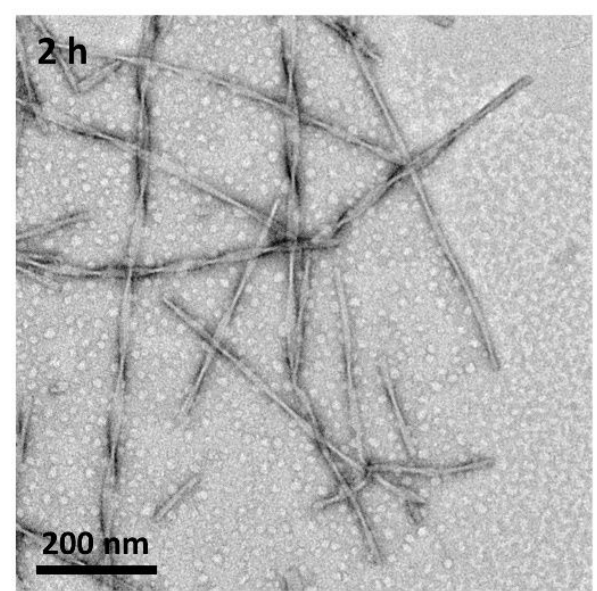

e)

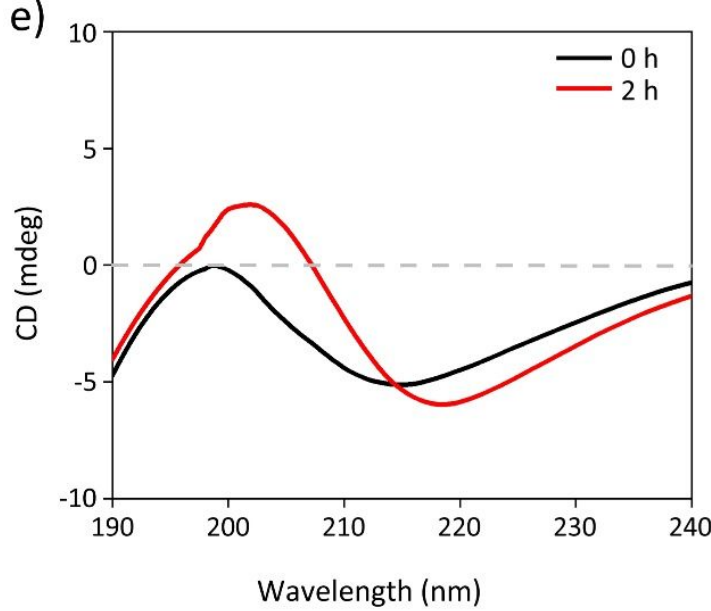

b)

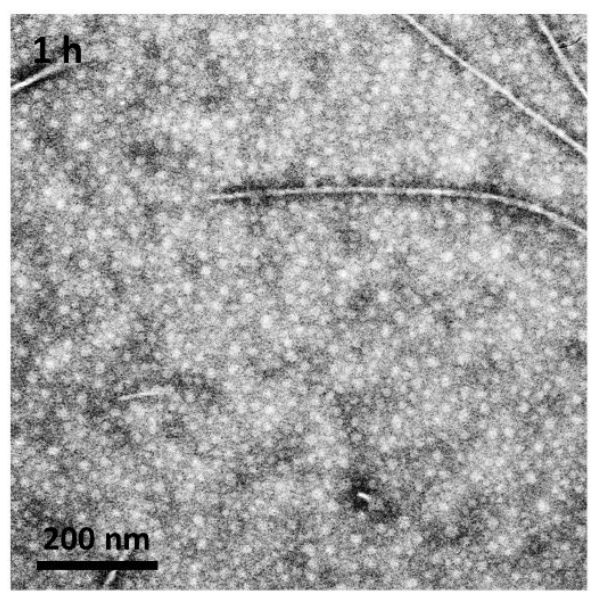

d)

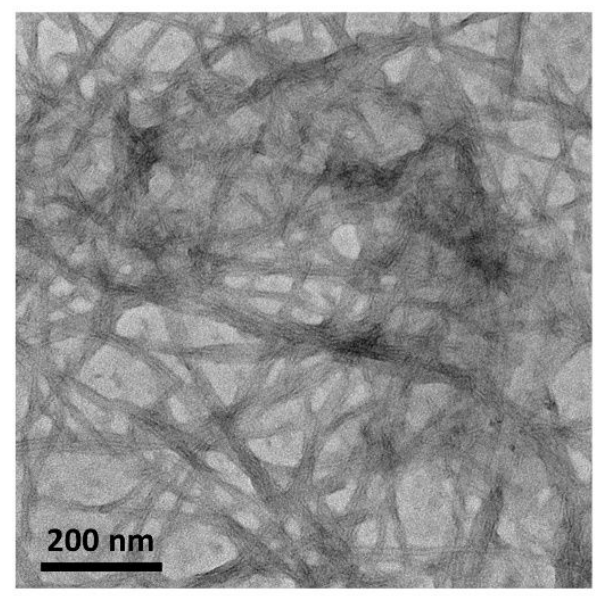

f)

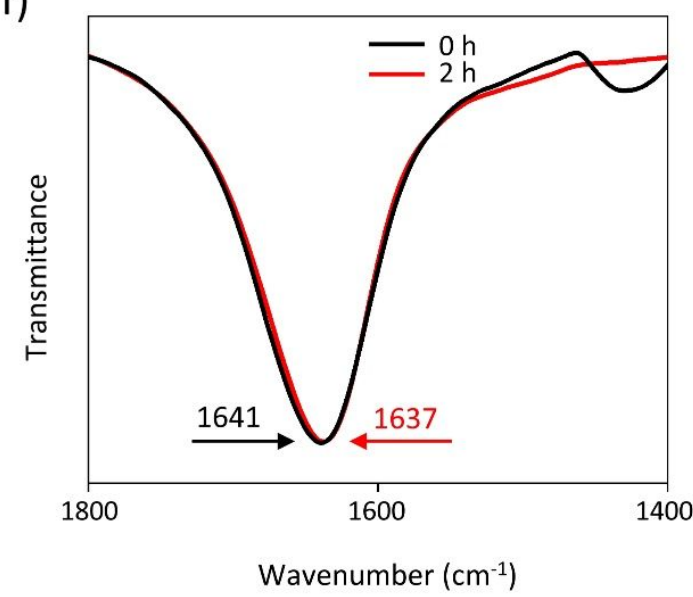

Fig. S2. Morphology transformation of LAFH NPs without incubated with $\mathrm{Ca}^{2+}$. a-d) TEM images of LAFH NPs without $\mathrm{Ca}^{2+}$ at $0 \mathrm{~h} \mathrm{(a),} 1 \mathrm{~h}(\mathrm{~b}), 2 \mathrm{~h}(\mathrm{c})$ and $4 \mathrm{~h}(\mathrm{~d})$ indicating the LAFH NPs could transform into fibrous structure without $\mathrm{Ca}^{2+}$ with lower speed. e) CD spectra and f) FT-IR spectra of LAFH NPs in the absence of $\mathrm{Ca}^{2+}$ in aqueous solution at different time point, indicating the formation of $\beta$-sheet structure. 
$\mathrm{Oh}$

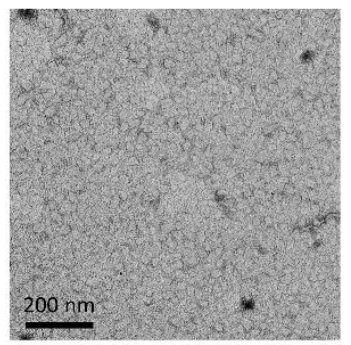

$1 \mathrm{~h}$

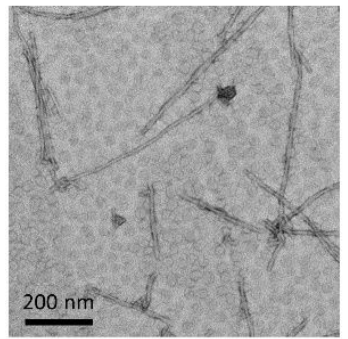

$2 \mathrm{~h}$

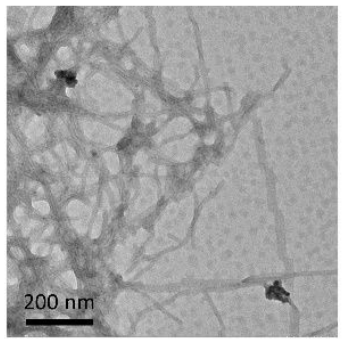

$4 \mathrm{~h}$

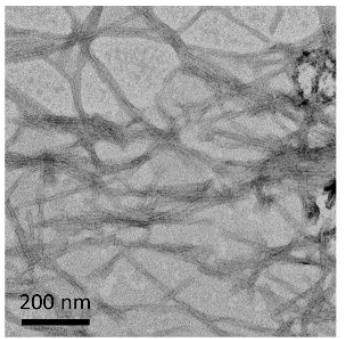

Figure S3. TEM images of LAFH $(20 \mu \mathrm{M})$ incubated with $\mathrm{Na}^{+}(\mathrm{NaCl}, 20 \mu \mathrm{M})$ at $0,1,2$ and $4 \mathrm{~h}$.

a)

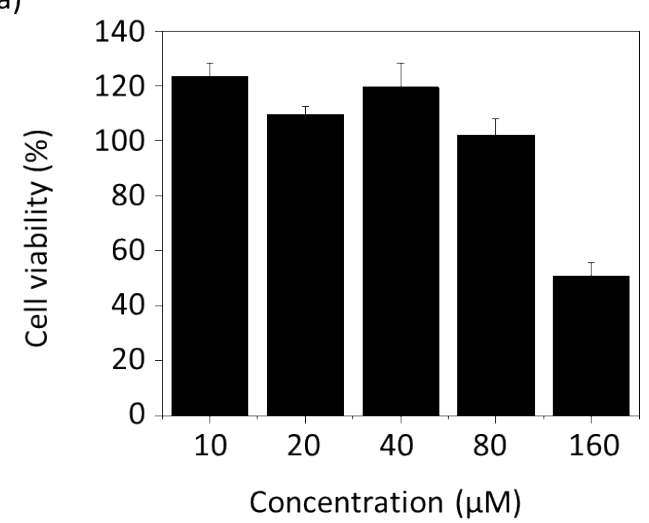

b)

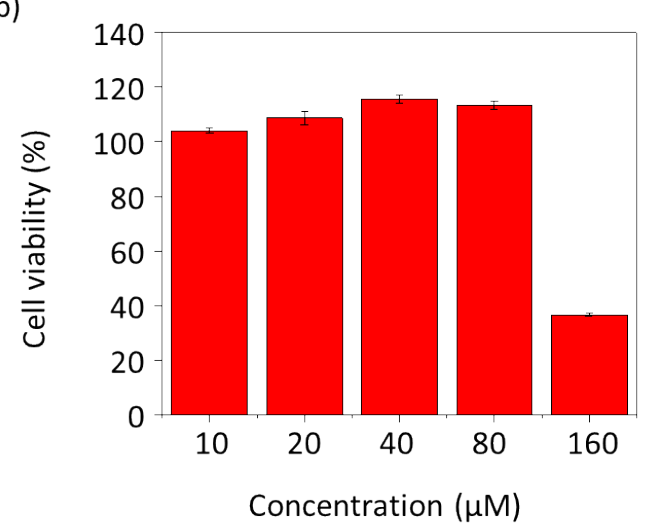

Fig. S4. Cell viability of a) HUVECs and b) MCF-7 cells to LAFH NPs by CCK-8 assay for 24 h, suggesting the negligible cytotoxicity of LAFH to HUVECs and MCF-7 cells below the concentration of $80 \mu \mathrm{M}$. 
LAFH
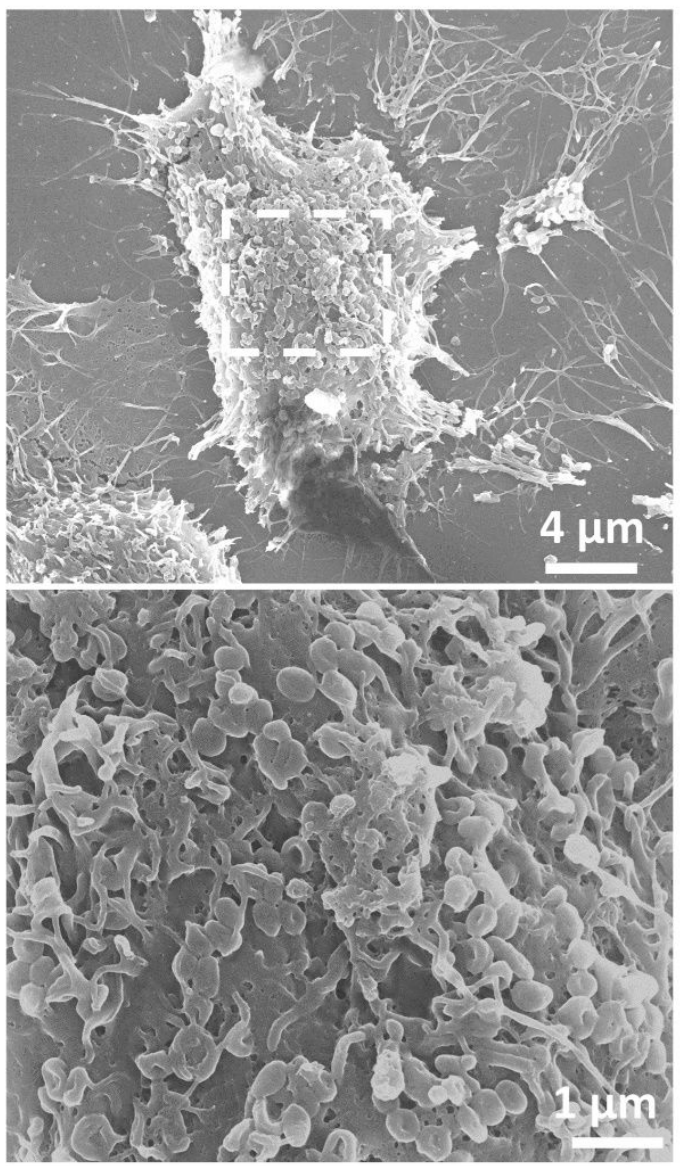

PBS
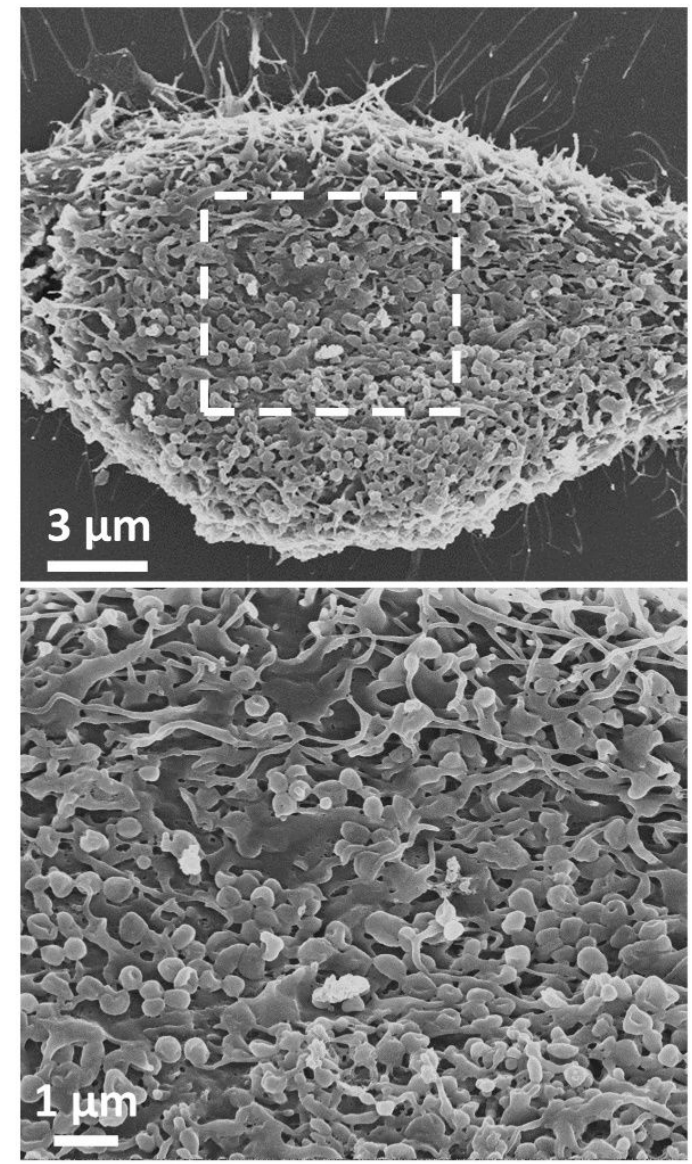

Figure S5. SEM images of LAFH NPs and PBS incubated MCF-7 cells. No obvious fibrous structure could be observed in the surface of LAFH or PBS treated MCF-7 cells. 
a)

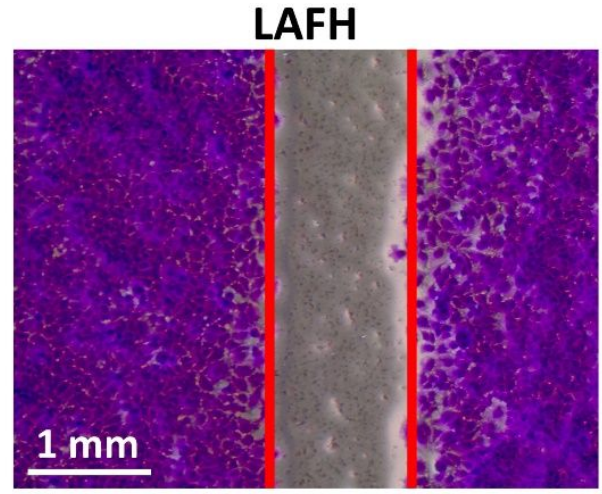

c)

e)
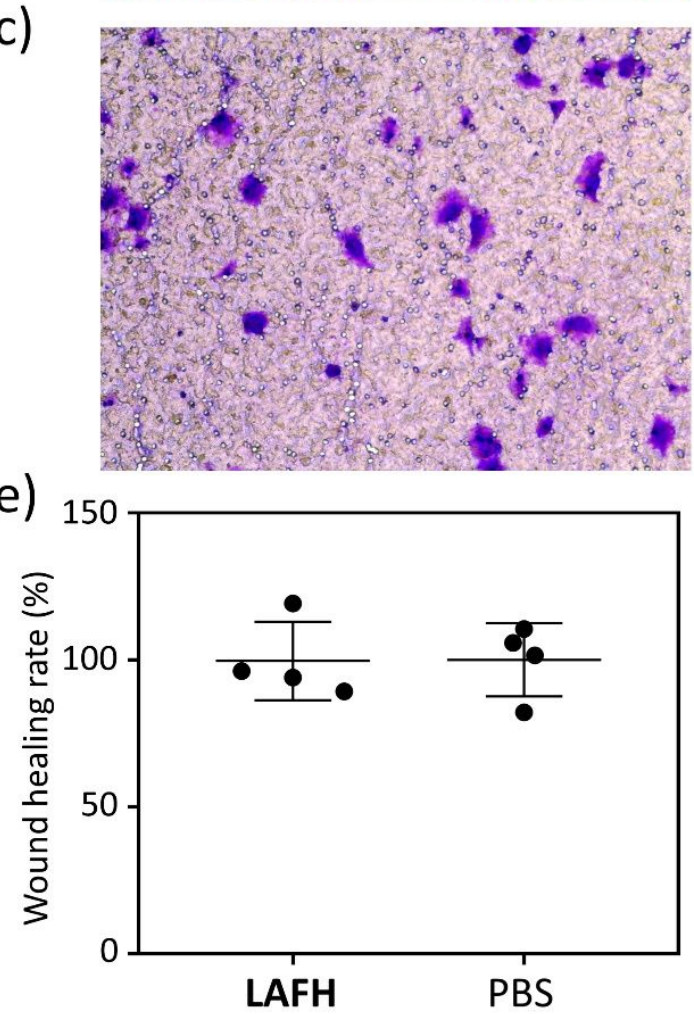

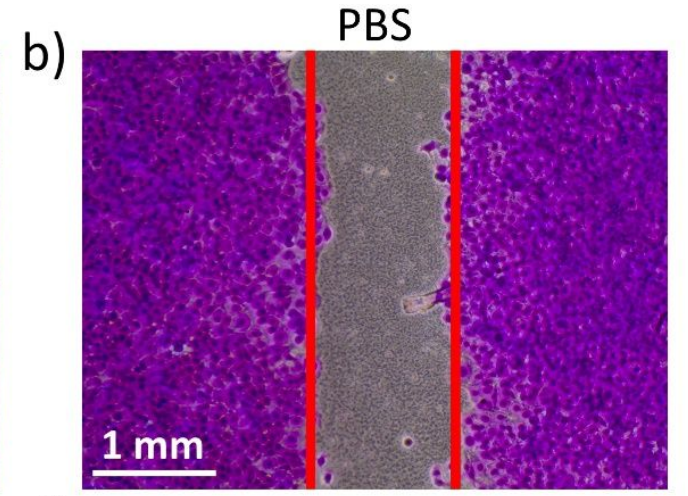

d)

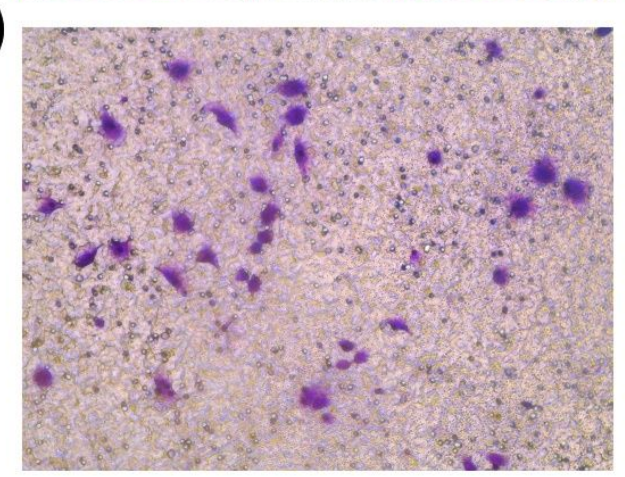

f)

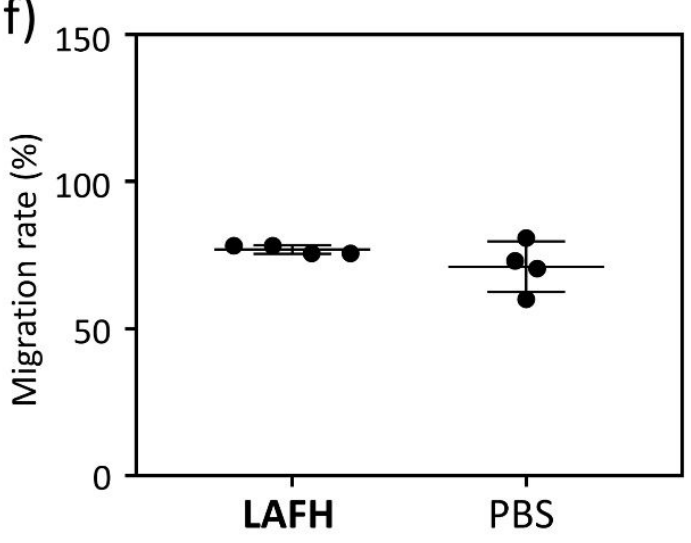

Figure S6. LAFH nanoparticles could not inhibit migration of MCF-7 cells. a-b) Microscopy image of wound healing experiment of MCF-7 cells treated with LAFH NPs (a) or PBS (b), white areas indicated no migrated areas. c-d) Microscopy image of transwell migration experiment of MCF-7 treated with LAFH NPs (c) or PBS (d), purple area indicated the migrated cells. e) Quantitative analysis of wound healing rate in $a-b$, no obvious difference in wound healing rate could be observed in LAFH or PBS group. f) Quantitative analysis of migration rate in c-d, no obvious difference in migration rate could be observed in LAFH or PBS group. 

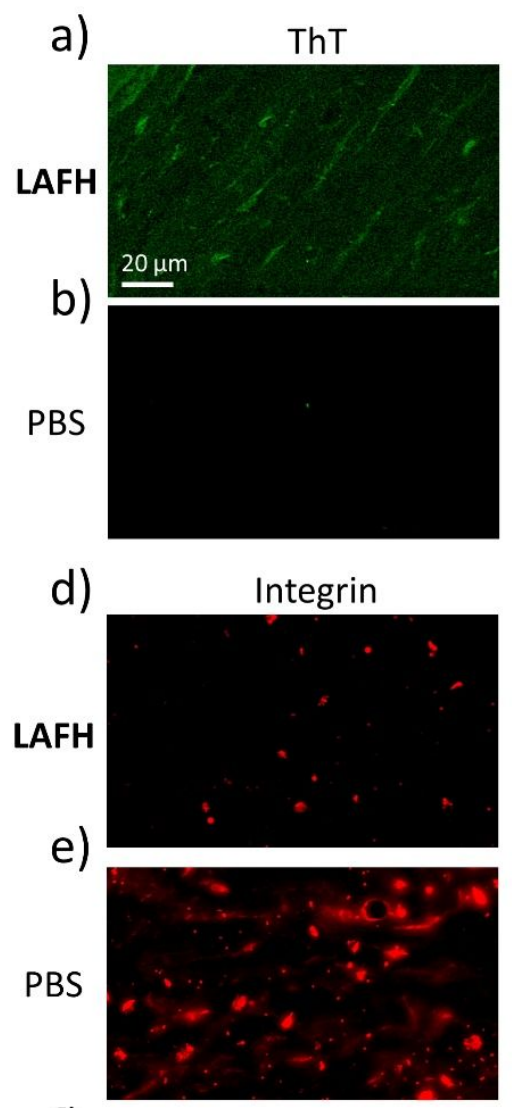

f)

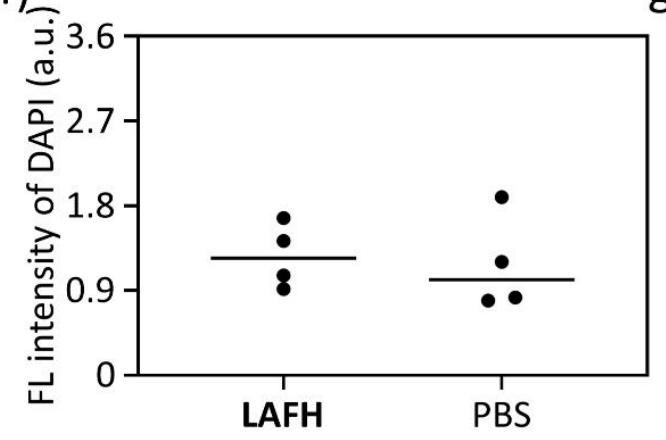

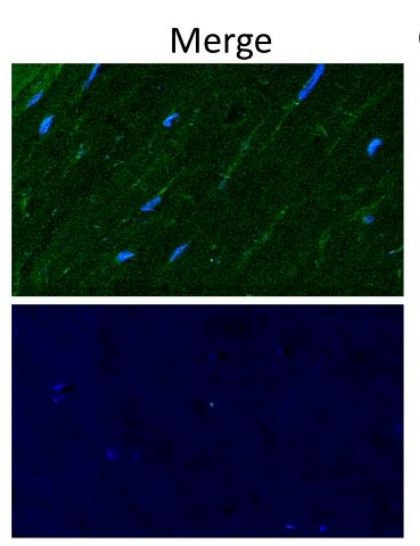
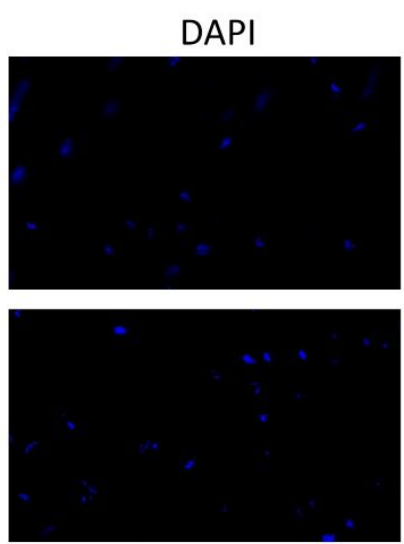

g) c)
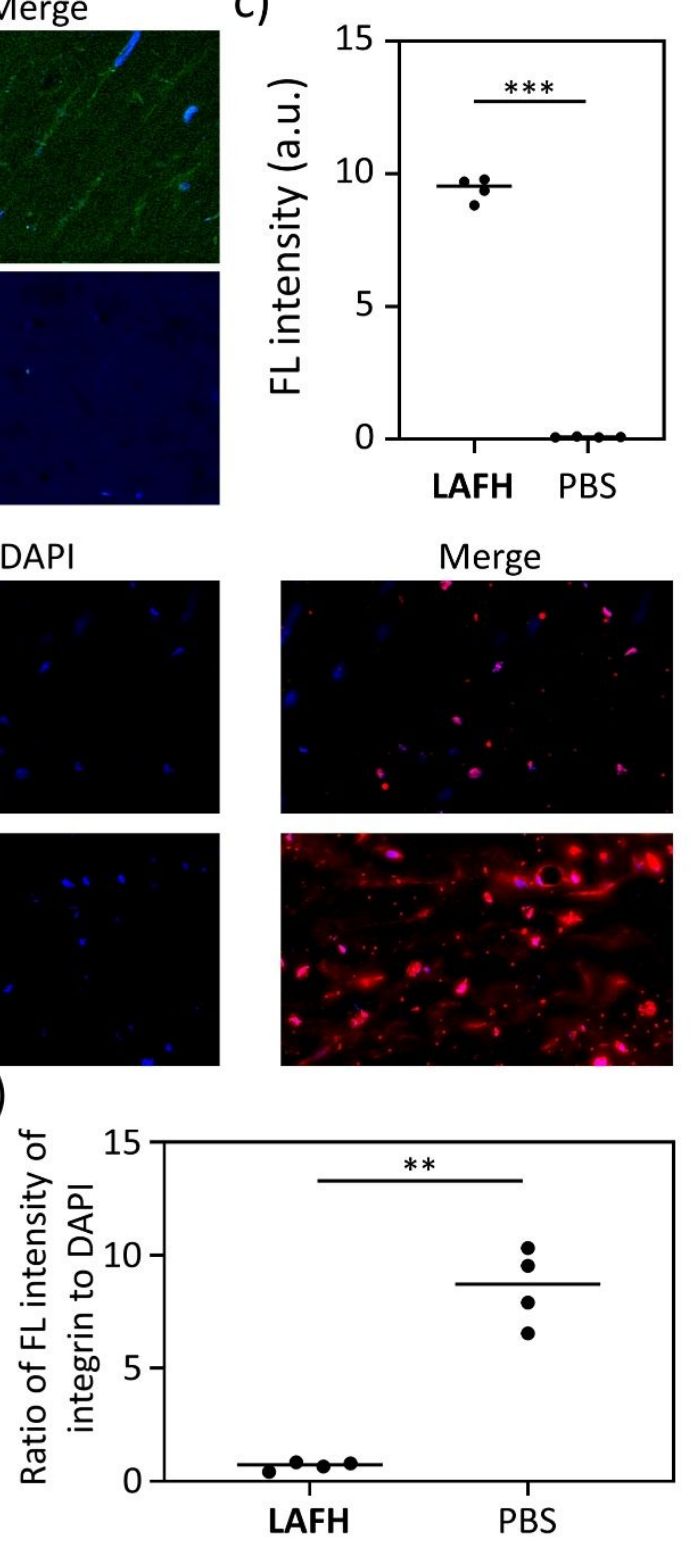

Figure S7. LAFH binds to $\alpha_{v} \beta_{3}$ with long-term retention in CNV rabbit model in 2 weeks in vivo (two injections on 0 and 7 day, respectively). a,b) Immunofluorescence images of fluorescence signal of CNV area after injection of LAFH NPs (a) or PBS (b) for 2 weeks. Green signal (ThT) for LAFH and blue signal for DAPI. c) Quantitative analysis of intensity of green fluorescence signal in (a) and (b), indicating the accumulation and retention of LAFH in 2 weeks. d,e) Immunofluorescence images of fluorescence signal of integrin in CNV area after injection of LAFH NPs (d) or PBS (e) for 2 weeks. Red signal for integrin $\alpha_{v} \beta_{3}$ and blue signal for DAPI. f,g) Quantitative analysis of fluorescence intensity of DAPI (f) and ratio of fluorescence intensity of integrin to DAPI (g) in (d) and (e), indicating the less detection of integrin $\alpha_{v} \beta_{3}$. 


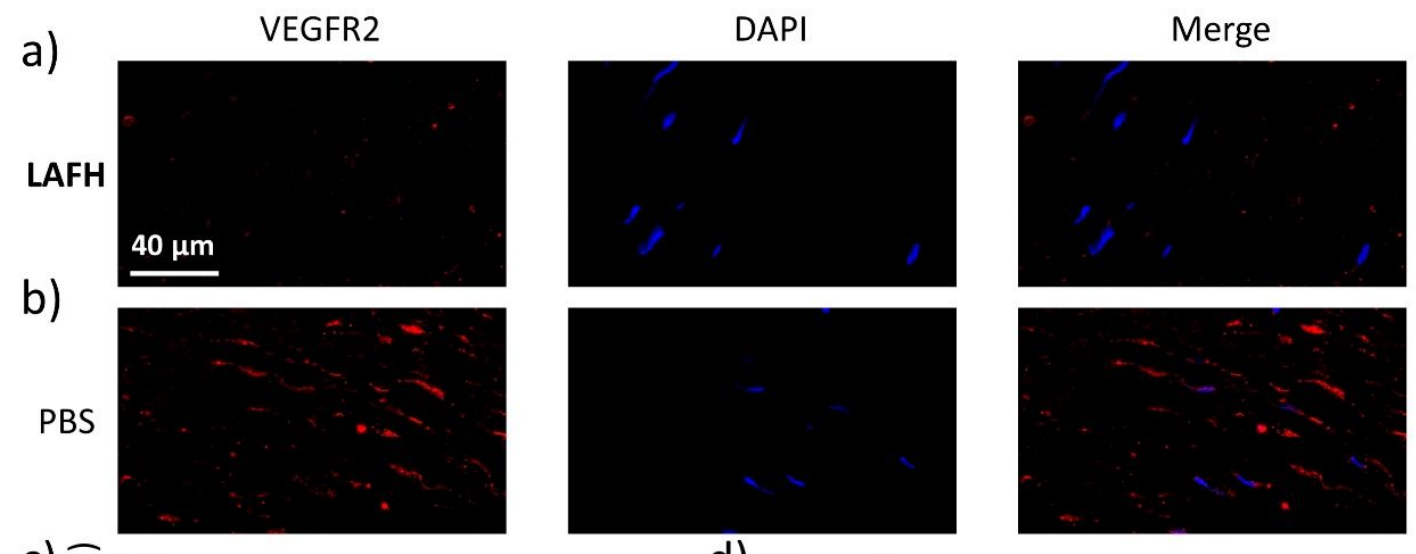

c)

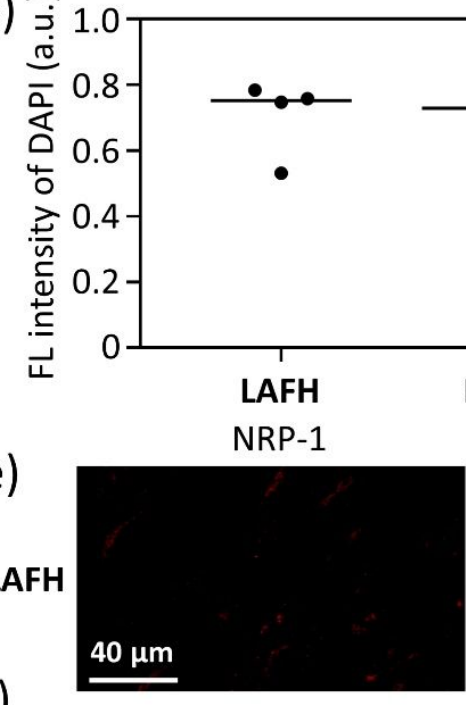

f)

PBS

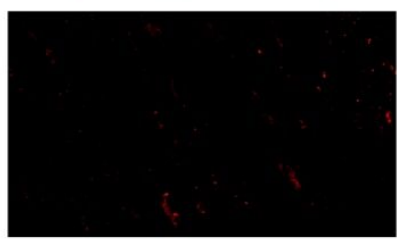

g)

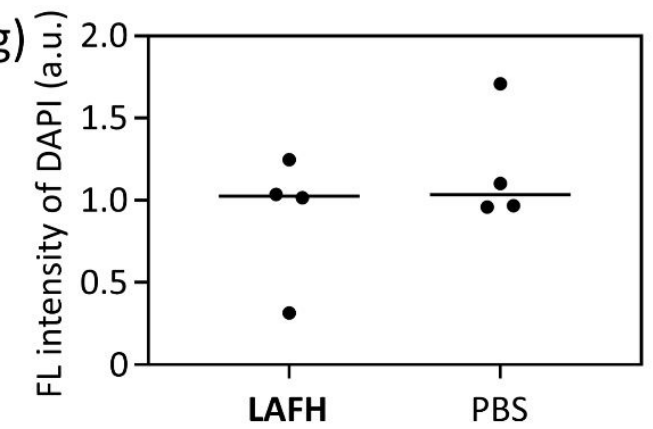

d)

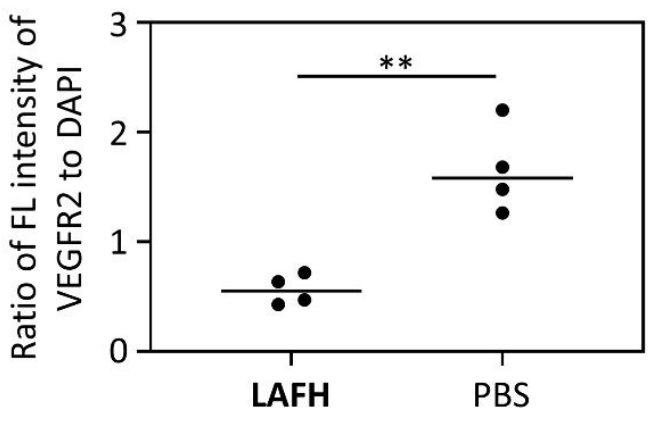

DAPI
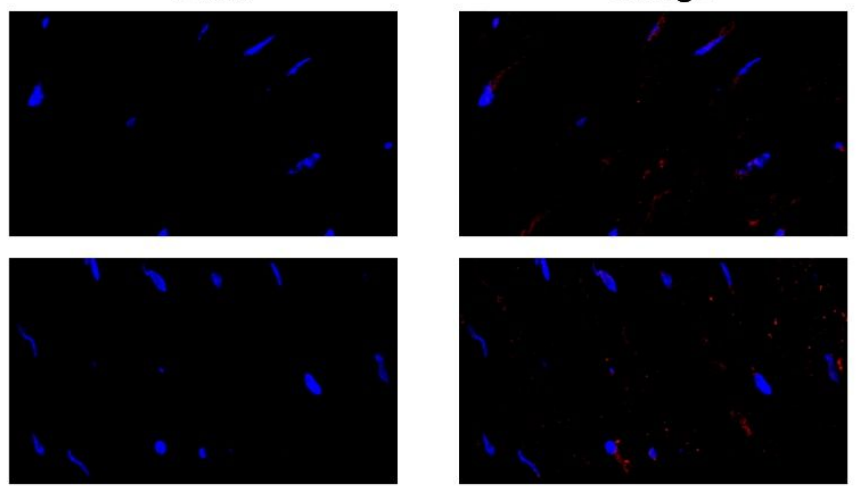

h) 4

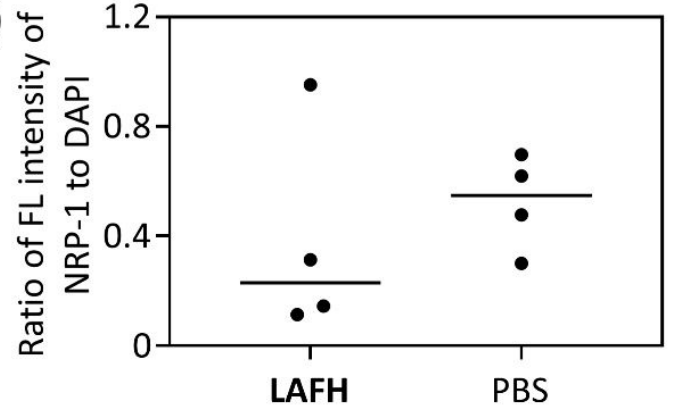

Figure S8. Coverage of LAFH nanofibers on ECs partially blocks VEGFR2 or NRP-1 in 2 weeks. a,b) Immunofluorescence images of fluorescence signal of VEGFR2 in CNV area after injection of LAFH NPs (a) or PBS (b) for 2 weeks. Red signal for VEGFR2 and blue signal for DAPI. c,d) Quantitative analysis of fluorescence intensity of DAPI (c) and ratio of fluorescence intensity of VEGFR2 to DAPI (d) in (a) and (b), indicating the less detection of VEGFR2 in CNV area. e,f) 
Immunofluorescence images of fluorescence signal of NRP-1 in CNV area after injection of LAFH NPs (e) or PBS (f) for 2 weeks. Red signal for NRP-1 and blue signal for DAPI. g,h) Quantitative analysis of fluorescence intensity of DAPI (g) and ratio of fluorescence intensity of NRP-1 to DAPI (h) in (e) and (f), indicating the less detection of NRP-1 in CNV area. 


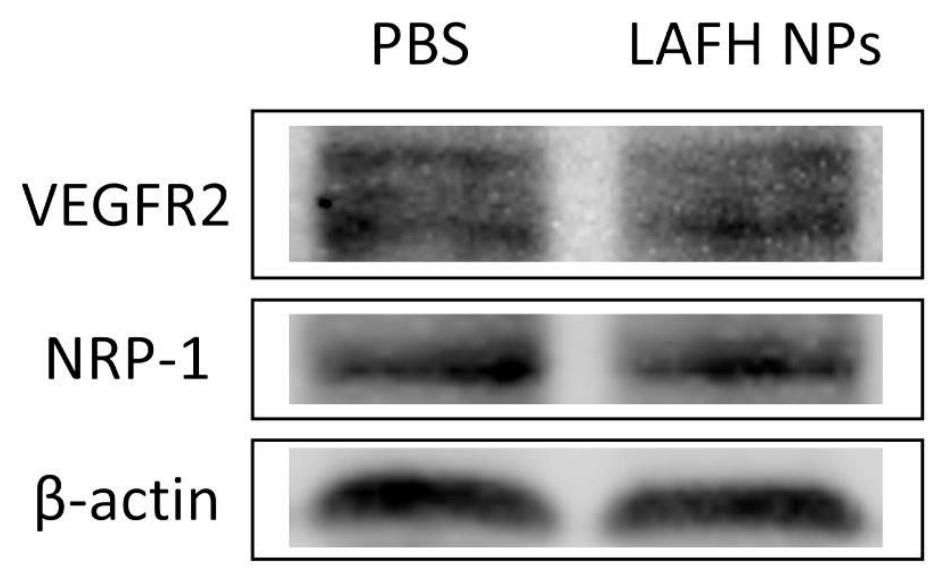

Figure 59. Western blot results of LAFH NPs or PBS treated HUVECs cells on the expression of VEGFR2 and NRP-1. 

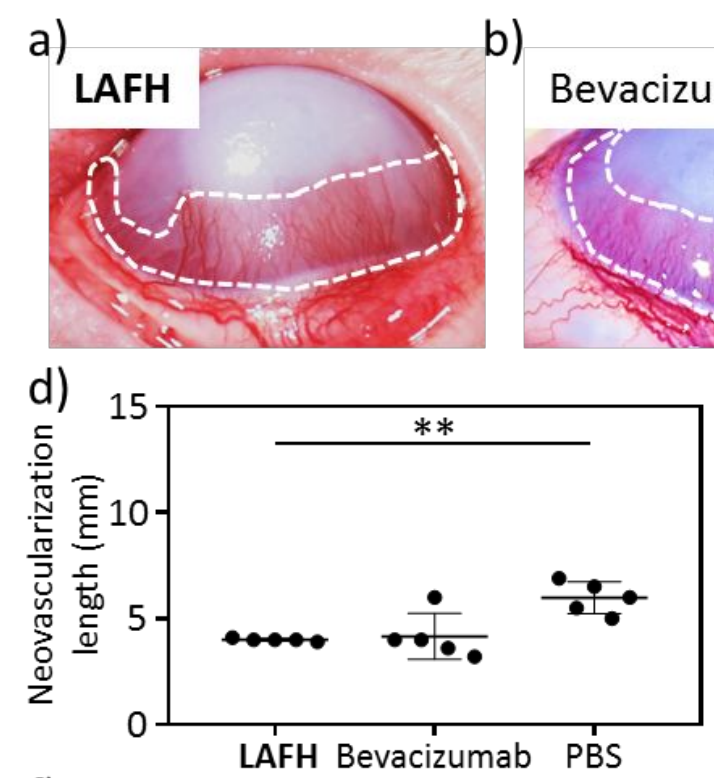

f)
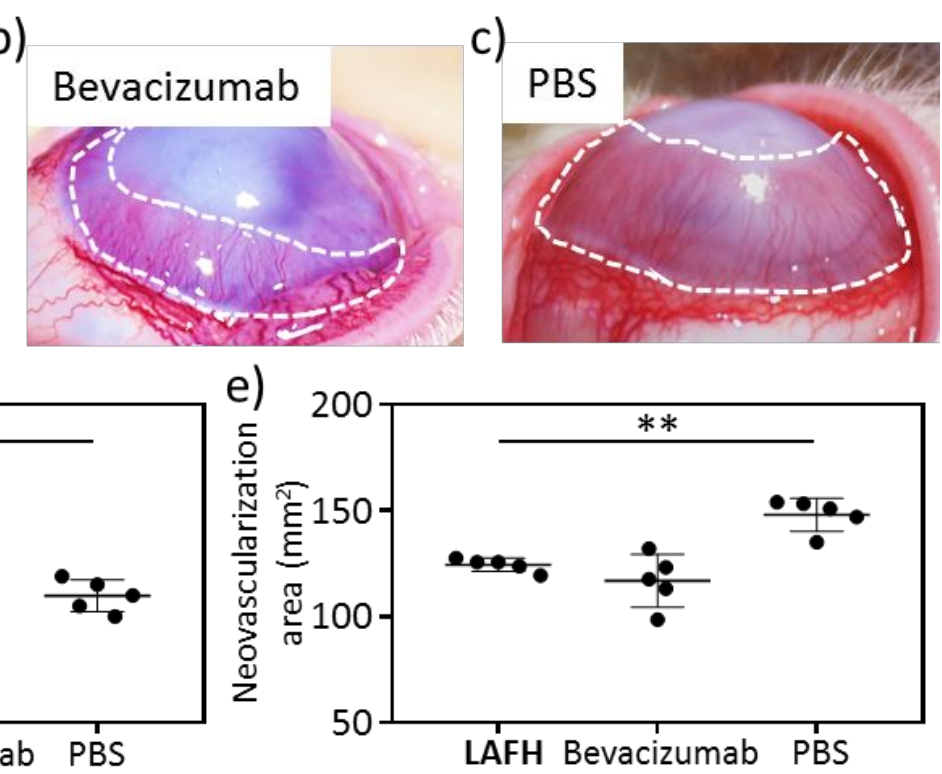

g)

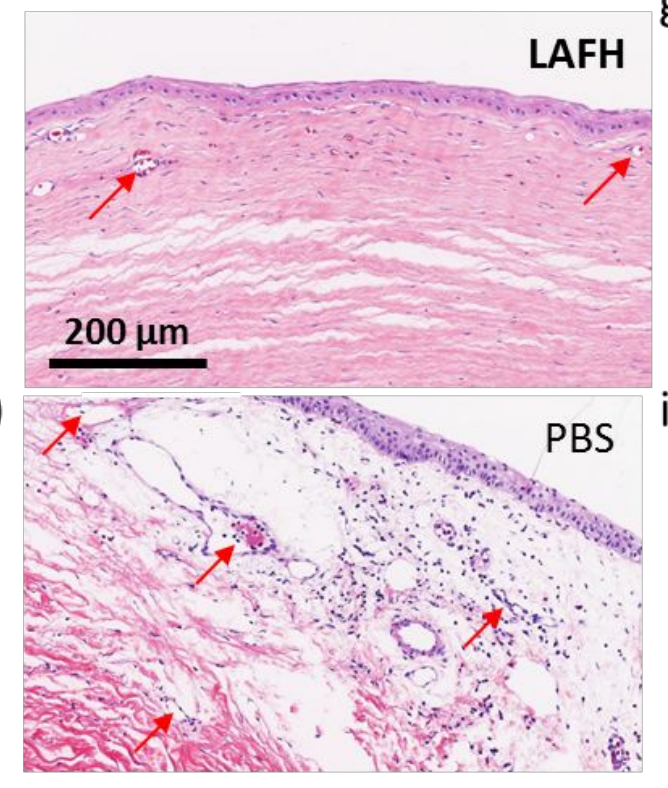

j)

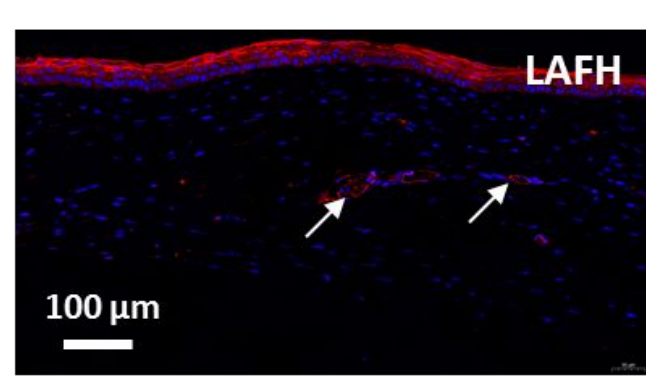

I)

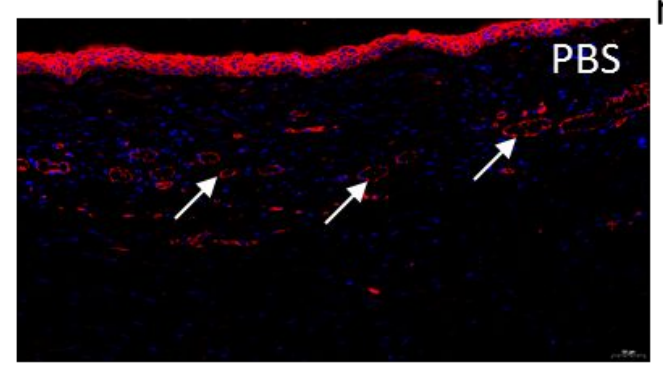

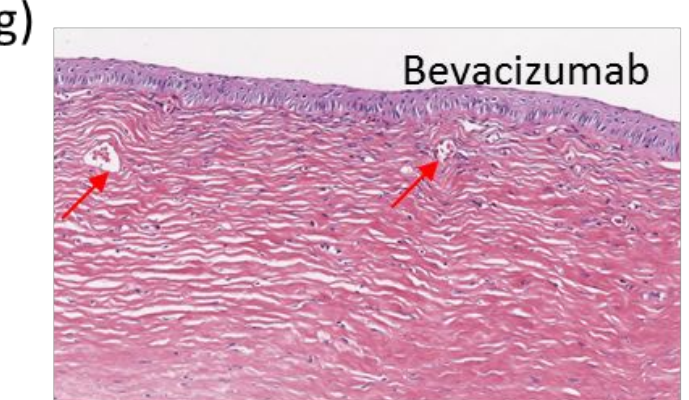

i)

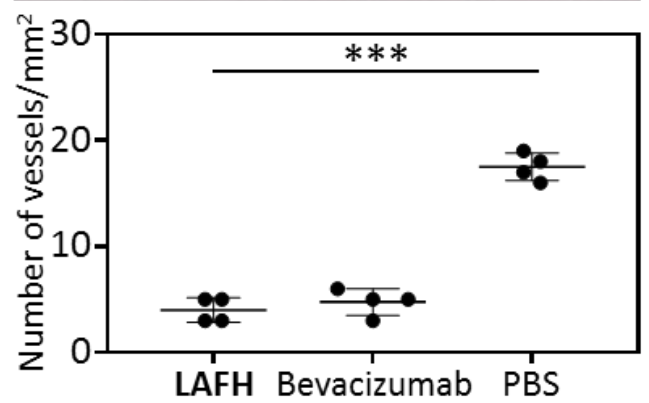

k)

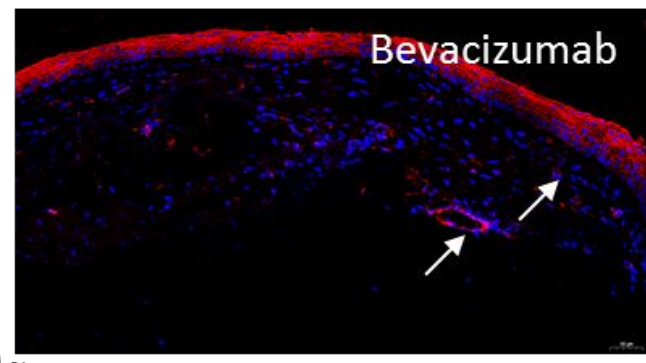

m)

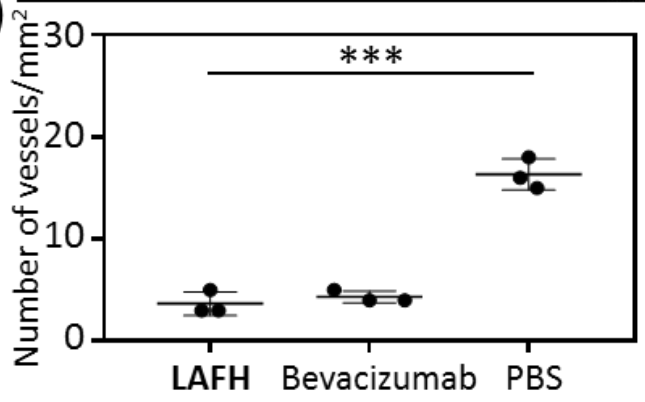


Figure S10. LAFH inhibits angiogenesis in CNV rabbit model in 2 weeks in vivo. a-c) Photo of eyes on in CNV rabbit model treated with LAFH NPs (a), bevacizumab (b) and PBS (c) for 2 weeks, respectively. White dash for neovascularization in CNV area. d,e) Quantitative analysis in the length (d) and area (e) of neovascularization in CNV area, suggesting the therapeutic effect of LAFH and bevacizumab comparing with PBS group. f-h) H\&E staining of eye cup in CNV rabbit model at day 14 after treatment of LAFH NPs (f), bevacizumab (g) and PBS (h). Red arrows for newly formed blood vessels. i) Quantitative analysis on the number of newly formed blood vessels in (f-h), indicating less new blood vessel formed after treatment of LAFH. $j-I)$ Immunofluorescence images of CNV area in LAFH NPs (j), bevacizumab (k) or PBS (I) treated CNV rabbit for 2 weeks. White arrows indicated the newly formed blood vessel. m) Quantitative analysis on the number of newly formed blood vessels in $(j-I)$, suggesting less new blood vessel formed after treatment of LAFH NPs. 